\title{
A Hadamard-type open map theorem for submersions and applications to completeness results in control theory
}

\author{
Andrea Bonfiglioli • Annamaria Montanari • \\ Daniele Morbidelli
}

Received: 9 September 2014 / Accepted: 17 December 2014 / Published online: 31 December 2014 (C) Fondazione Annali di Matematica Pura ed Applicata and Springer-Verlag Berlin Heidelberg 2014

\begin{abstract}
We prove a quantitative openness theorem for $C^{1}$ submersions under suitable assumptions on the differential. We then apply our result to a class of exponential maps appearing in Carnot-Carathéodory spaces, and we improve a classical completeness result by Palais.
\end{abstract}

Keywords Hadamard inverse map theorem - Submersion - Complete vector fields . Involutive vector fields $\cdot$ Moore-Penrose pseudoinverse

Mathematics Subject Classification Primary 17B66; Secondary 34H05 - 58C25

\section{Introduction}

It is well known by the Hadamard global inverse map theorem that $a C^{1}$ local diffeomorphism $f: \mathbb{R}^{N} \rightarrow \mathbb{R}^{N}$ with $\sup _{\mathbb{R}^{N}}\left|d f(x)^{-1}\right|<\infty$ is in fact a global diffeomorphism. Instead, if we consider a submersion $f: \mathbb{R}^{N} \rightarrow \mathbb{R}^{n}$ with $N>n$, one can not expect injectivity, but it is reasonable to hope that suitable conditions on the differential $d f(x)$ may ensure that the map $f$ is onto. This has been discussed in very general setting in the recent paper [20].

The first purpose of this paper is to prove a quantitative openness result for $C^{1}$ submersions, which in particular gives a surjectivity theorem assuming uniform openness of the differential map. Our second task is to apply such result to a class of exponential maps appearing in the

\footnotetext{
A. Bonfiglioli · A. Montanari $(\varangle) \cdot$ D. Morbidelli

Dipartimento di Matematica, Università degli Studi di Bologna, Piazza di Porta San Donato, 5, 40126 Bologna, Italy

e-mail: annamaria.montanari@unibo.it

A. Bonfiglioli

e-mail: andrea.bonfiglioli6@unibo.it

D. Morbidelli

e-mail: daniele.morbidelli@unibo.it
} 
analysis of Carnot-Carathéodory spaces. This will enable us to obtain an improvement of a classical completeness result for vector fields originally due to Palais [18].

Here is our first result.

Theorem 1.1 Let $M$ be a (finite dimensional) manifold of class $C^{2}$ and let $g$ be a $C^{1}$ Riemannian metric on $M$. Assume that we are given positive constants $C_{0}<\infty$ and $r_{0} \leq \infty, a$ point $x_{0} \in \mathbb{R}^{q}$ and a map $f: B_{\mathrm{Euc}}\left(x_{0}, r_{0}\right) \rightarrow M$ which is $C^{1}$ regular on the Euclidean ball $B_{\text {Euc }}\left(x_{0}, r_{0}\right) \subset \mathbb{R}^{q}$ such that the differential (tangent map) of $f$ satisfies

$$
d f(x)\left(B_{\text {Euc }}\left(0, C_{0}\right)\right) \supseteq\left\{\xi \in T_{f(x)} M: g(\xi, \xi) \leq 1\right\} \text { for all } x \in B_{\text {Euc }}\left(x_{0}, r_{0}\right) .
$$

Then

$$
f\left(B_{\text {Euc }}\left(x_{0}, r_{0}\right)\right) \supseteq B_{\operatorname{Rie}}\left(f\left(x_{0}\right), \frac{r_{0}}{2 C_{0}}\right) .
$$

In the statement $T_{z} M$ is the tangent space to $M$ at $z$ and $B_{\text {Rie }}$ denotes the ball in $M$ with respect to the Riemannian distance generated by $g$.

Note that the theorem holds true with $r_{0}=\infty$ and takes the following form: if $f: \mathbb{R}^{q} \rightarrow$ $(M, g)$ is a $C^{1}$ submersion and it satisfies (1.1) for all $x \in \mathbb{R}^{q}$, then the map $f$ is onto. We observe that, as it will appear in the proof of Theorem 1.1, our techniques also apply to the case when the source space is a Riemannian manifold, but we do not pursue this issue here. On a concrete level, we will read (1.1) as a uniform bound of the Moore-Penrose pseudoinverse of $d f(x)$ (see the discussion in Sect. 2.1).

Topological properties of a submersion $f$ between Riemannian manifolds have been discussed by Hermann [5], who exploited the notion of horizontal path lifting, requiring that $f \in C^{1,1}$ and that $\left.d f(x)\right|_{\operatorname{ker} d f(x)^{\perp}}$ is an isometry. Earle and Eells [3] generalized Hermann's condition to the infinite-dimensional setting, showing that horizontal path-liftings can be constructed under the assumption that $d f(x)$ admits a Lipschitz-continuous family $s(x)$ of right inverses. It must be said that the mentioned papers are not primarily concerned with quantitative openness or surjectivity, but they are more focused on the topological/differential structure of the fibers of $f$. More recent results are due to Rabier [20], who, in order to have surjectivity of a $C^{1,1}$ submersion $f$ from infinite-dimensional Finsler manifolds, requires the existence of a Lipschitz-continuous right inverse satisfying a suitable global upper bound. This somewhat forces the assumption $f \in C^{1,1}$. The only reference dealing with $C^{1}$ maps (between linear spaces only) is [19]. That paper does not rely on path liftings and it does not seem to provide the quantitative estimates (1.2) of Theorem 1.1.

In this paper we shall work in finite dimension, but without requiring the $C^{1,1}$ regularity, because in the applications simple examples show that our submersions are at most $C^{1, \alpha}$ for some $\alpha<1$ (see the discussion before the statement of Theorem 1.2). In this case, horizontal liftings are not necessarily unique, see Example 2.1 below.

Next we discuss how we are able to apply Theorem 1.1 to Control Theory. According to a remarkable result of Palais [18], given a family $\mathcal{H}=\left\{X_{1}, \ldots, X_{m}\right\}$ of complete vector fields on a manifold $M$ generating a finite-dimensional Lie algebra $\mathfrak{g}$, any vector field $Y \in \mathfrak{g}$ is complete. Recall that we say that a vector field $Z$ on a manifold $M$ is complete if for each $x \in M$ the integral curve $t \mapsto e^{t Z} x$ is defined for $\left.t \in\right]-\infty,+\infty$ [. For the sake of brevity, we shall refer to this result as Palais' Completeness Theorem.

Our aim is to provide an improvement of this theorem, in that we consider lower regularity assumptions on the vector fields and, most importantly, we replace finite dimensionality with the following involutivity hypothesis: There is $s \in \mathbb{N}$ such that the family $Y_{1}, \ldots, Y_{q}$ of the nested commutators [see (3.1)] of length at most $s$ of the original vector fields of $\mathcal{H}$ satisfies 


$$
\left[Y_{i}, Y_{j}\right](x)=\sum_{k=1}^{q} c_{i, j}^{k}(x) Y_{k}(x), \quad \text { for all } i, j=1, \ldots, q \quad x \in M,
$$

for suitable functions $c_{i, j}^{k}$ globally bounded on $M$. In the sequel, we will say that $\mathcal{H}$ is $s$-involutive if (1.3) holds true.

Our approach is different from Palais' and from the approaches of the existing alternative proofs of Palais' Completeness Theorem. At our knowledge, all existing proofs of the Palais Theorem rely on ideas similar to each other, making a strong use the Third Theorem of Lie; see [2, p. 145], [9, p. 966], [18, p. 96], [21, p. 147]. Our more general assumptions, which allow the coefficients $c_{i, j}^{k}$ to be non-constant, prevent us from applying the Third Theorem of Lie to the Lie algebra generated by $\left\{X_{1}, \ldots, X_{m}\right\}$, which may not be finite dimensional (see Example 3.4).

Let $\mathcal{H}=\left\{X_{1}, \ldots, X_{m}\right\}$ be a given family of complete vector fields on $M$ and assume that the involutivity condition (1.3) holds for some $s \geq 1$. Note that requiring that the coefficients $c_{i, j}^{k}$ are constant on $M$ (a particular case of our assumptions) means that Lie $\mathcal{H}$ is finite dimensional. Look at the non-autonomous Cauchy problem

$$
\dot{\gamma}=\sum_{k=1}^{q} b_{k}(t) Y_{k}(\gamma) \text { for a.e. } t \quad \gamma(0)=x \in M,
$$

where $\|b\|_{L^{\infty}(\mathbb{R})} \leq 1$. We will show that there are $\varepsilon, \delta>0$ and vector fields $Z_{1}, \ldots, Z_{v} \in \mathcal{H}$ such that for all $x \in M, T \in[0, \varepsilon]$ one can find $t_{1}, \ldots, t_{v} \in \mathbb{R}$ such that we can write

$$
\gamma(T)=e^{t_{\nu} Z_{\nu}} \cdots e^{t_{1} Z_{1}} x \quad \text { with the estimate } \sum_{j=1}^{v}\left|t_{j}\right| \leq \delta .
$$

This statement will be made precise in the language of Carnot-Carathéodory geometry. Since the choice of $\varepsilon, \delta$ and $Z_{1}, \ldots, Z_{v}$ is independent of $x \in M$, an iteration of (1.4) will prove that $\gamma(T)$ is defined for all $T \in]-\infty,+\infty$ [. This will yield the completeness of $\gamma$, thus providing the mentioned improvement of Palais' Completeness Theorem.

The sequence of exponentials appearing in (1.4) will be organized as a composition of approximate exponential maps, which now we informally describe. Let $\mathcal{H}=\left\{X_{1}, \ldots, X_{m}\right\}$ be our given family of vector fields of class $C^{s}$ on a $C^{s+1}$ manifold $M$ of dimension $n$ and assume that $\mathcal{H}$ satisfies the $s$-involutivity hypothesis (1.3). Let $\exp _{\text {ap }}\left(Y_{j}\right) x$ be the approximate exponential of the nested commutator $Y_{j}$ in (3.1), see, e.g., [14]. To fix the ideas, if $Y_{j}=$ $\left[X_{k}, X_{\ell}\right], t \geq 0$ and $x \in M$, then

$$
\exp _{\mathrm{ap}}\left(t Y_{j}\right) x:=\exp _{\mathrm{ap}}\left(t\left[X_{k}, X_{\ell}\right]\right) x:=e^{-\sqrt{t} X_{\ell}} e^{-\sqrt{t} X_{k}} e^{\sqrt{t} X_{\ell}} e^{\sqrt{t} X_{k}} x .
$$

(The construction of the family $Y_{1}, \ldots, Y_{q}$ is described in detail at the beginning of Sect. 3.1.) The definition can be generalized to $t<0$ and to commutators of higher length, using more complicated compositions of elementary exponentials. This will be precisely defined in Sect. 3.2.

Introduce the map $E_{x}: \mathbb{R}^{q} \rightarrow M$,

$$
E_{x}(h):=\exp _{\mathrm{ap}}\left(h_{1} Y_{1}\right) \circ \cdots \circ \exp _{\mathrm{ap}}\left(h_{q} Y_{q}\right) x .
$$

Note that the point $E_{x}(h)$ is actually of the form appearing in (1.4), although the number of variables $q$ can be much larger than the dimension of $M$. Using a first-order expansion for the tangent map of $E_{x}$, we will recognize that the map $E_{x}$ is a submersion from a neighborhood of the origin to a suitable submanifold of $M$ (the Sussmann orbit of the system $\mathcal{H}$ 
containing $x$ ). Moreover, if the target manifold is equipped with a suitable Riemannian metric, $E$ satisfies the quantitative assumptions of Theorem 1.1. Concerning regularity, in [13, Example 5.7]) it is shown that we can expect at most that $E \in C^{1, \alpha}$ for some $\alpha<1$. Thus we obtain the following:

Theorem 1.2 Let $\mathcal{H}=\left\{X_{1}, \ldots, X_{m}\right\}$ be a family of complete vector fields of class $C^{s}$ on a manifold $M$ of class $C^{s+1}$ and assume that $\mathcal{H}$ is $s$-involutive [i.e., it satisfies (1.3)]. Let $\rho$ be the Carnot-Carathéodory distance associated with the vector fields $Y_{1}, \ldots, Y_{q}$. If the functions $c_{i, j}^{k}$ in (1.3) are globally bounded on $M$, then there are absolute constants $\varepsilon, \delta>0$ such that

$$
E_{x}\left(B_{\text {Euc }}(0, \varepsilon)\right) \supseteq B_{\rho}(x, \delta) \text { for all } x \in M \text {. }
$$

In the language of Carnot-Carathéodory analysis, Theorem 1.2 can be referred to as a ball-box theorem. The new feature here is the uniformity of the constants $\varepsilon, \delta$ with respect to $x \in M$, which can be proved as a consequence of the global boundedness of the coefficients in (1.3). From a technical point of view, the novelty is that we are using submersions instead of local diffeomorphism (which are usually obtained by a restriction of $E_{x}$ to linear subspaces whose choice is quite delicate). Ultimately, our arguments, although they do not provide injectivity results, seem to be simpler than previous ones (compare to [13,17]).

Our improved version of Palais' Completeness Theorem follows as a corollary.

Corollary 1.3 (Improved Palais' theorem) Under the hypotheses of Theorem 1.2, the Cauchy problem

$$
\dot{\gamma}=\sum_{j=1}^{q} b_{j}(t) Y_{j}(\gamma) \text { a.e. with } \gamma(0)=x
$$

has global solution for all $x \in M$ and for each $b \in L_{\text {loc }}^{\infty}(\mathbb{R})$.

A solution to the non-autonomous Cauchy problem (1.7) is a continuous path $\gamma$ on $[0, T]$, which solves the integral equation

$$
\gamma(t)=x+\int_{0}^{t} \sum_{j} b_{j}(s) Y_{j}(\gamma(s)) d s \text { for all } t \in[0, T] .
$$

Local existence and uniqueness follow from standard theory (e.g., the Picard iteration scheme). It turns out that $\gamma$ is absolutely continuous and the ODE in (1.7) holds for a.e. $t$. Moreover, in spite of the irregularity of the coefficients $b_{j}$, since the vector fields $Y_{j}$ are $C^{1}$, the classical results on dependence on initial data hold and it turns out that the solution depends in a $C^{1}$ way from the initial data $x$. This can be seen by carefully checking that the proof for autonomous systems (see [8, Chapter 17]) also works for a non-autonomous system like (1.7).

Before closing this introduction, we mention some applications of Palais' Completeness Theorem (these applications also give a motivation for our investigation of this remarkable result), which is often applied jointly with Palais' Integrability Theorem (as referred to, e.g., in [1]): The latter theorem states that, given a family of complete vector fields generating a finite-dimensional Lie algebra $\mathfrak{g}, \mathfrak{g}$ can be integrated to a global action of a Lie group. Palais' Completeness/Integrability Theorems naturally intervene in the study of Lie transformation groups of geometric structures on a manifold, see [2, Theorem H]. They also find many applications in Control Theory: 
- see the controllability results in [7, Section 3], furnishing explicit characterizations for the reachable sets;

- see the feedback-stabilization results for ODE systems in [16, Theorem 3.1];

- see [9, Theorems 5.1 and 6.1], furnishing a characterization of the class of control systems that are globally diffeomorphic to a linear system (on a Lie group or a homogeneous space) in terms of finite-dimensional Lie algebras of complete vector fields (this is precisely the assumption in Palais' Completeness Theorem).

Furthermore, let us also mention that Palais' Theorem finds applications in the study of ordinary/stochastic differential equations (see e.g., [11,15]). We finally remark that Palais' Completeness and Integrability Theorems have been recently generalized also to the framework of infinite-dimensional algebras/manifolds, see [1,12].

\section{Open map theorem for submersions}

\subsection{Linear algebra preliminaries}

Fact 1. Let $V, W$ be finite-dimensional linear spaces and let $g$ be an inner product on $V$. If $T: V \rightarrow W$ is a linear map and $T(V)=W$, then there is a unique Moore-Penrose pseudoinverse map $T^{\dagger}: W \rightarrow V$ such that for each $w \in W, T^{\dagger}(w)=v_{\mathrm{LN}}$ is the minimal norm solution (here 'LN' stands for 'least norm') of the system $T(v)=w$. The solution $v_{\mathrm{LN}}$ is characterized by the conditions

$$
T\left(v_{\mathrm{LN}}\right)=w \quad \text { and } \quad v_{\mathrm{LN}} \perp \operatorname{ker} T .
$$

In particular, $T^{\dagger}$ is a right inverse of $T$, i.e. $T T^{\dagger}=I_{W}$.

Fact 2. If $p \leq q$ and $A \in \mathbb{R}^{p \times q}$ has independent rows, then the Moore-Penrose pseudoinverse $A^{\dagger}$ is a right inverse of $A$ and has the explicit form

$$
A^{\dagger}=A^{T}\left(A A^{T}\right)^{-1} \text {. }
$$

For each $y \in \mathbb{R}^{p}$ the minimal norm solution $x_{\mathrm{LN}}$ of the system $A x=y$ has the form $x=A^{\dagger} y$ and, among all solutions of such system, it is characterized by the orthogonality condition $x_{\mathrm{LN}} \perp \operatorname{ker} A$.

Fact 3. If $v_{1}, \ldots, v_{q}$ span a $p$-dimensional linear space $W$, then we can define a natural inner product $g$ on $W$ by letting for all $v, v^{\prime} \in W$

$$
g\left(v, v^{\prime}\right):=\left\langle T^{\dagger} v, T^{\dagger} v^{\prime}\right\rangle_{\mathbb{R}^{q}},
$$

where $T^{\dagger}$ is the pseudoinverse of the linear map $T: \mathbb{R}^{q} \rightarrow W$, defined by $T(\xi)=$ $\sum_{j=1}^{q} v_{j} \xi_{j}$. If $p=q$, then $T^{\dagger}=T^{-1}$ and the vectors $v_{1}, \ldots, v_{p}$ turn out to be orthonormal. Note that $g$ is nondegenerate because $T T^{\dagger}=I_{W}$.

Fact 4. If $A$ is a matrix with independent rows, we have $\left|A^{\dagger}\right|^{-1}=\min _{|y|=1}\left|A^{T} y\right|$. Indeed, let $n \leq N$ and let $A \in \mathbb{R}^{n \times N}$. First, it is well known that $A$ is onto if and only if $A^{T}$ is oneto-one (just because $\left.\operatorname{Im} A=\left(\operatorname{ker} A^{T}\right)^{\perp}\right)$. The required equality can be checked as follows. First observe that, since $A$ has full rank and $n \leq N, A A^{T}$ is positive-definite, and moreover, 
$A^{\dagger}=A^{T}\left(A A^{T}\right)^{-1}$. Thus,

$$
\begin{aligned}
\left|A^{\dagger}\right|^{2} & =\max _{|y|=1}\left|A^{T}\left(A A^{T}\right)^{-1} y\right|^{2}=\max _{|y|=1}\left\langle A^{T}\left(A A^{T}\right)^{-1} y, A^{T}\left(A A^{T}\right)^{-1} y\right\rangle \\
& =\max _{|y|=1}\left\langle\left(A A^{T}\right)^{-1} y, y\right\rangle=\lambda_{\max }\left(\left(A A^{T}\right)^{-1}\right)=\frac{1}{\lambda_{\min }\left(A A^{T}\right)},
\end{aligned}
$$

where $\lambda_{\max }$ and $\lambda_{\min }$ denote the largest/smallest eigenvalue. On the other hand,

$$
\min _{|y|=1}\left|A^{T} y\right|^{2}=\min _{|y|=1}\left\langle A A^{T} y, y\right\rangle=\lambda_{\min }\left(A A^{T}\right) .
$$

Thus Fact 4 is checked.

\subsection{Proof of Theorem 1.1}

Here we prove Theorem 1.1. We will use a horizontal path-lifting argument choosing the Moore-Penrose pseudoinverse as a right inverse of the differential. Indeed, the requirement (1.1) is equivalent to the assumption that $d f(x): T_{x} \mathbb{R}^{q} \rightarrow T_{f(x)} M$ is onto for all $x \in$ $B_{\text {Euc }}\left(x_{0}, r_{0}\right)$ and that the estimate ${ }^{1}\left|d f(x)^{\dagger}\right| \leq C_{0}$ holds true for all $x \in B_{\text {Euc }}\left(x_{0}, r_{0}\right)$, where $\left|d f(x)^{\dagger}\right|$ denotes the operator norm of the Moore-Penrose pseudoinverse of $d f(x)$.

Proof of Theorem 1.1 Assume that $x_{0}=0$ and denote briefly $B_{0}:=B_{\mathrm{Euc}}\left(0, r_{0}\right) \subset \mathbb{R}^{q}$. Let $y \in B_{\text {Rie }}\left(f(0), \frac{r_{0}}{2 C_{0}}\right)$. Thus there is $r_{1}<\frac{r_{0}}{2 C_{0}}$ and an absolutely continuous path $\gamma:[0,1] \rightarrow$ $M$ such that

$$
\gamma(0)=f(0),|\dot{\gamma}(t)|_{g} \leq r_{1} \text { for a.e.t, and } \gamma(1)=y .
$$

Our strategy relies on the construction of a lifting of $\gamma$. Since there is no uniqueness, it is crucial to make a "minimal" choice (think to the example $f: \mathbb{R}^{2} \rightarrow \mathbb{R}$ with $f\left(x_{1}, x_{2}\right)=$ $\left.x_{1}+\arctan x_{2}\right)$. Precisely, we claim that there is an absolute continuous path $\theta:[0,1] \rightarrow B_{0}$ such that

$$
\begin{aligned}
& \theta(0)=0, \\
& f(\theta(t))=\gamma(t) \quad \text { for all } t \in[0,1], \\
& \dot{\theta}(t) \perp \operatorname{ker} d f(\theta(t)) \quad \text { for a.e. } t \in[0,1] .
\end{aligned}
$$

We refer to the last line as a minimality condition. We remark that in the literature such condition is also called horizontality condition. Note that if $[a, b] \subset[0,1]$ and an absolutely continuous path $\theta:[a, b] \rightarrow B_{0}$ satisfies (2.2b) and (2.2c), then, differentiating, we get

$$
|\dot{\theta}(t)|=\left|d f(\theta(t))^{\dagger} \dot{\gamma}(t)\right| \leq C_{0} r_{1} \text { for a.e. } t \in[a, b] .
$$

Thus, $\theta$ is Lipschitz continuous with Lipschitz constant $C_{0} r_{1}$.

Let

$$
\begin{gathered}
\bar{b}:=\sup \left\{b \in[0,1] \text { such that exists } \theta \in \operatorname{Lip}\left([0, b], B_{0}\right) \text { with } \theta(0)=0\right. \\
f \circ \theta=\gamma \text { on }[0, b] \text { and } \dot{\theta} \perp \operatorname{ker} d f(\theta) \text { a.e. on }[0, b]\} .
\end{gathered}
$$

Our aim is to prove that $\bar{b}=1$.

Step 1. We first show that $\bar{b}>0$.

$\overline{1}$ An equivalent assumption is $\inf _{|\xi|=1}\left|d f(x)^{T} \xi\right| \geq C_{0}^{-1}$ for all $x$. See the linear algebra Fact 4 in Sect. 2.1. 
Fix coordinates $\xi: U \rightarrow V$ from an open set $U \subset M$ containing $f(0)$ to an open set $V \subset \mathbb{R}^{p}$. Let $\Omega \subset B_{0}$ be the connected component of $f^{-1}(U)$ containing the origin. Let $\psi:=\xi \circ f: \Omega \rightarrow V$ and $\sigma(t)=(\xi \circ \gamma)(t)$ for all $t \in[0,1]$ such that $\gamma(t) \in U$. Denote by $J_{\psi}(\theta) \in \mathbb{R}^{p \times q}$ the Jacobian matrix of $\psi$ at the point $\theta \in \Omega$ and look at the Cauchy problem

$$
\dot{\theta}(t)=J_{\psi}(\theta(t))^{\dagger} \dot{\sigma}(t) \text { a.e., with } \theta(0)=0 .
$$

Here $J_{\psi}(\theta)^{\dagger} \in \mathbb{R}^{q \times p}$ denotes the Moore-Penrose inverse of $J_{\psi}(\theta)$. Since $J_{\psi}(\theta)$ has full rank $p \leq q$, we have the explicit formula

$$
J_{\psi}(\theta)^{\dagger}=J_{\psi}(\theta)^{T}\left(J_{\psi}(\theta) J_{\psi}(\theta)^{T}\right)^{-1} \text { for all } \theta \in \Omega,
$$

which shows that the function $\theta \mapsto J_{\psi}(\theta)^{\dagger}$ is continuous. Therefore, the Cauchy problem (2.5) has at least a solution $\theta:[0, b[\rightarrow \Omega$ on some small nonempty interval $[0, b[$. More precisely, $\theta$ is absolutely continuous and solves the integral equation

$$
\theta(t)=\int_{0}^{t} J_{\psi}(\theta(\tau))^{\dagger} \dot{\sigma}(\tau) d \tau \text { for all } t \in[0, b[.
$$

At any differentiability point $t$ we have

$$
J_{\psi}(\theta(t)) \dot{\theta}(t)=J_{\psi}(\theta(t)) J_{\psi}(\theta(t))^{\dagger} \dot{\sigma}(t)=\dot{\sigma}(t),
$$

which implies that $\psi \circ \theta=\sigma$ on $\left[0, b\left[\right.\right.$. Applying $\xi^{-1}$ we get $f \circ \theta=\gamma$.

Finally, to accomplish Step 1, we check the orthogonality condition. Since $\theta$ solves the problem (2.5), we have for almost all $t$ the condition $\dot{\theta}(t) \perp \operatorname{ker} J_{\psi}(\theta(t))$. By the chain rule $d \psi(\theta)=d \xi(f(\theta)) d f(\theta)$ and since $d \xi(y)$ is an invertible linear map for all $y \in U$, we see that $\operatorname{ker} J_{\psi}(\theta(t))=\operatorname{ker} d f(\theta(t))$, as required.

Step 2. We show that $\bar{b}=1$.

Assume by contradiction that $\bar{b}<1$. Let $b_{n} \uparrow \bar{b}$ and let $\theta_{n}:\left[0, b_{n}\right] \rightarrow B_{0}$ be a Lipschitzcontinuous path such that $\theta_{n}(0)=0$,

$$
d f\left(\theta_{n}\right) \dot{\theta}_{n}=\dot{\gamma} \quad \text { and } \quad \dot{\theta}_{n} \perp \operatorname{ker} d f\left(\theta_{n}\right) \text { a.e. on }\left[0, b_{n}\right] .
$$

We have in fact $\dot{\theta}_{n}=\left(d f\left(\theta_{n}\right)\right)^{\dagger} \dot{\gamma}(t)$ a.e. on $\left[0, b_{n}\right]$. Therefore, by (2.3) we get the estimate

$$
\left|\theta_{n}(t)\right| \leq C_{0} r_{1}|t| \leq \frac{r_{0}}{2},
$$

because we know that $r_{1}<\frac{r_{0}}{2 C_{0}}$. Therefore the image of $\theta_{n}$ is strictly inside the ball $B_{0}$. Next, extend each $\theta_{n}$ on the whole interval $[0, \bar{b}]$ by letting $\theta_{n}(t)=\theta_{n}\left(b_{n}\right)$ for $t \in\left[b_{n}, \bar{b}\right]$. The family $\theta_{n}$ is uniformly bounded and Lipschitz continuous [see (2.3)]. Thus, by Ascoli-Arzelà theorem, up to extracting a subsequence, we may assume that $\theta_{n}$ converges uniformly on $[0, \bar{b}]$ to a limit function $\theta$. Start from the integral formula

$$
\theta_{n}(t)=\int_{0}^{t} d f\left(\theta_{n}(\tau)\right)^{\dagger} \dot{\gamma}(\tau) d \tau \quad \forall t \in\left[0, b_{n}\right],
$$

and note that coordinate manifestations such as (2.6) show that $\theta \mapsto d f(\theta)^{\dagger}$ is continuous. Passing to the limit as $n \rightarrow+\infty$ (by dominated convergence), we discover that $\theta$ satisfies (2.2a), (2.2b) and (2.2c) on $[0, \bar{b}]$ and has global Lipschitz constant $C_{0} r_{1}$ [again by (2.3)].

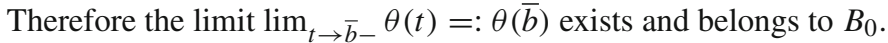

To conclude Step 2, fix coordinates $\xi^{\prime}: U^{\prime} \rightarrow V^{\prime} \subset \mathbb{R}^{p}$ on a neighborhood $U^{\prime}$ of $f(\theta(\bar{b})$ ) and, arguing as in Step 1, construct for some $\varepsilon>0$ a Lipschitz solution $\Theta:\left[\bar{b}, \bar{b}+\varepsilon\left[\rightarrow B_{0}\right.\right.$ 
such that

$\Theta(\bar{b})=\theta(\bar{b}), \quad d f(\Theta(t)) \dot{\Theta}(t)=\dot{\gamma}(t)$ and $\dot{\Theta}(t) \perp \operatorname{ker} d f(\Theta(t))$ for a.e. $t \in[\bar{b}, \bar{b}+\varepsilon]$.

Thus we have extended the solution $\theta$ on an interval strictly larger than $[0, \bar{b}]$ and we get a contradiction. This concludes the proof of Step 2 and, jointly, the proof of the theorem.

Example 2.1 We show that the horizontal lifting of a path via a $C^{1}$ lifting is not unique. Consider the $C^{1}$ submersion $f: \mathbb{R}^{3} \rightarrow \mathbb{R}$

$$
f\left(x_{1}, x_{2}, x_{3}\right)=\frac{2}{3} x_{1}\left|x_{1}\right|^{1 / 2}+\frac{2}{3} x_{2}\left|x_{2}\right|^{1 / 2}+x_{3},
$$

whose gradient is $\nabla f(x)=\left(\left|x_{1}\right|^{1 / 2},\left|x_{2}\right|^{1 / 2}, 1\right)$. Both the paths

$$
\theta(t)=\left(\frac{1}{4} t^{2}, 0, t\right) \text { and } \phi(t)=\left(0, \frac{1}{4} t^{2}, t\right)
$$

are liftings of the same path $f \circ \theta=f \circ \phi$. Moreover, they are minimal, because they are both integral curves of the continuous vector field $\nabla f$.

Example 2.2 The "horizontal bundle" $(\operatorname{ker} d f)^{\perp}$ is in general not involutive. For example, taking $f(x, y, z)=(x+y z, y) \in \mathbb{R}^{2}$, one sees that $(\operatorname{ker} d f)^{\perp}$ is generated by the vector fields $\partial_{y}$ and $\partial_{x}+z \partial_{y}+y \partial_{z}$, which form a non-involutive system.

\section{Applications to completeness results in control theory}

\section{$3.1 s$-Involutive families of vector fields}

Let $X_{1}, \ldots, X_{m}$ be a family of vector fields on an $n$-dimensional manifold $M$ and let $s \in \mathbb{N}$. Assume that $M$ is $C^{s+1}$ and that $X_{j} \in C^{s}(M)$ for $j=1, \ldots, m$. Fix a bijection between the finite set of words $\left\{w=w_{1} w_{2} \cdots w_{\ell}: w_{j} \in\{1, \ldots, q\}, \ell \in\{1, \ldots, m\}\right\}$ and the set $\{1, \ldots, q\}$ where $q \in \mathbb{N}$ is suitable. Enumerate accordingly as $Y_{1}, \ldots, Y_{q}$ all the nested commutators up to length $s$ of the original vector fields $X_{1}, \ldots, X_{m}$. Precisely, for each $j \in\{1, \ldots, q\}, Y_{j}$ is a bracket of the type

$$
Y_{j}=\left[X_{w_{1}},\left[X_{w_{2}}, \ldots,\left[X_{w_{\ell-1}}, X_{w_{\ell}}\right] \ldots\right]\right],
$$

where $w_{1}, \ldots, w_{\ell} \in\{1, \ldots, m\}$. In such case we say that $\ell$ is the length of $Y_{j}$. Note that each $Y_{j}$ is a $C^{1}$ vector field.

As in [14], we say that $\mathcal{H}$ is $s$-involutive if $X_{j} \in C^{s}(M)$ for all $j$ and there are locally bounded functions $c_{i, j}^{k}$ (with $1 \leq i, j, k \leq q$ ) so that

$$
\left[Y_{i}, Y_{j}\right](x)=\sum_{k=1}^{q} c_{i, j}^{k}(x) Y_{k}(x) \text { for all } i, j \in\{1, \ldots, q\} .
$$

Here, in local coordinates we have $Y_{j}=\sum_{\alpha=1}^{n} Y_{j}^{\alpha} \partial_{\alpha}$ and $Y_{j}^{\alpha} \in C^{1}$. Furthermore we set $\left[Y_{i}, Y_{j}\right]=\sum_{\alpha}\left(Y_{i} Y_{j}^{\alpha}-Y_{j} Y_{i}^{\alpha}\right) \partial_{\alpha}$, which is a continuous vector field. Observe also that if $\operatorname{dim}(\operatorname{Lie} \mathcal{H})<\infty$, then the family $\mathcal{H}$ is involutive for some $s$ and the functions $c_{i, j}^{k}$ can be chosen to be constant. Moreover, the local boundedness of $c_{i, j}^{k}$ in (3.2) ensures that the distribution generated by the vector fields $Y_{j}$ is integrable, by Hermann's Theorem [6]. Thus 
$M$ can be decomposed as a disjoint union of orbits. Namely, we can define the orbit containing $x \in M$ as

$$
\mathcal{O}_{x}:=\left\{e^{t_{1} Z_{1}} \ldots e^{t_{v} Z_{v}} x: v \in \mathbb{N}, Z_{1}, \ldots, Z_{v} \in \mathcal{H} \text { and }\left(t_{1}, \ldots, t_{v}\right) \in \Omega_{Z_{1}, \ldots, Z_{v}, x}\right\},
$$

where $\Omega_{Z_{1}, \ldots, Z_{v}, x} \subset \mathbb{R}^{v}$ is a suitable (maximal) open set containing the origin in $\mathbb{R}^{v}$ such that all the exponential maps are well defined. Each orbit $\mathcal{O}$, equipped with a suitable topology (see [14]), is an immersed submanifold of class $C^{2}$ of $M$ and it is an integral manifold of the distribution generated by the vector fields $Y_{j}$. In other words, $T_{y} \mathcal{O}=\operatorname{span}\left\{Y_{j}(y): 1 \leq\right.$ $j \leq q\}$ for all $y \in \mathcal{O}$. The number $p_{x}:=\operatorname{dim}\left(\operatorname{span}\left\{Y_{j}(x): 1 \leq j \leq s\right\}\right)$ can be different at different points, but it is constant on a fixed orbit.

By the linear algebra preliminaries in the previous section, for any $y \in \mathcal{O}$ we can define an inner product $g$ on $T_{y} \mathcal{O}$ via the matrix $\left[Y_{1}(y), \ldots, Y_{q}(y)\right]$ as in (2.1). It can be checked that this gives a $C^{1}$ Riemannian metric on the $C^{2}$ manifold $\mathcal{O}$. The corresponding norm of $Z \in T_{y} \mathcal{O}$ is

$$
|Z|_{y}:=g(Z, Z)^{1 / 2}=\min \left\{|\xi|: \sum_{j=1} Y_{j}(y) \xi_{j}=Z\right\}=\left|\left[Y_{1}(y), \ldots, Y_{q}(y)\right]^{\dagger} Z\right|_{\mathrm{Euc}}
$$

Note that $|Z|_{y} \leq 1$ if and only if $Z$ is subunit in the Fefferman-Phong sense with respect to the family $Y_{1}, \ldots, Y_{q}$ (see [4]). We denote by $\rho(x, y)$ the distance associated with such a Riemannian metric, which is nothing but the Carnot-Carathéodory distance associated with the family of vector fields $Y_{1}, \ldots, Y_{q}$. Precisely, $\rho(x, y)$ is the infimum of all $T \geq 0$ such that one can find an absolutely continuous solution $\gamma:[0, T] \rightarrow M$ of the problem $\dot{\gamma}=\sum_{j} b_{j}(t) Y_{j}(\gamma)$ a.e., with $\gamma(0)=x, \gamma(T)=y$ and $\sum_{j} b_{j}^{2}(t) \leq 1$ a.e. Clearly, $\rho(x, y)<\infty$ for each pair of points on the same orbit.

We introduce the following notation for multi-indexes. Let $p, q \in \mathbb{N}$ with $1 \leq p \leq q$. Define

$$
\left.\mathcal{I}(p, q):=\left\{\left(i_{1}, \ldots, i_{p}\right): 1 \leq i_{1}<i_{2} \cdots<i_{p} \leq q\right)\right\} .
$$

Moreover, if $x \in M, p=p_{x}$ and $I \in \mathcal{I}(p, q)$ we let

$$
Y_{I}(x):=Y_{i_{1}}(x) \wedge \cdots \wedge Y_{i_{p}}(x) \in \bigwedge_{p} T_{x} M .
$$

Note that $Y_{I}(x) \neq 0$ means that $Y_{i_{1}}(x), \ldots, Y_{i_{p}}(x)$ are independent. Therefore, they generate $T_{x} \mathcal{O}_{x}$, because $p=p_{x}$ is its dimension. Moreover, for each $K=\left(k_{1}, \ldots, k_{p}\right) \in$ $\mathcal{I}(p, q)$ we have $Y_{k_{1}}(x), \ldots, Y_{k_{p}}(x) \in T_{x} \mathcal{O}$ and thus $Y_{k_{1}} \wedge \cdots \wedge Y_{k_{p}}$ is a scalar multiple of $Y_{i_{1}} \wedge \cdots \wedge Y_{i_{p}}$ at $x$. Since the set $\mathcal{I}(p, q)$ is finite, at any point $x \in M$, there is $I \in \mathcal{I}(p, q)$ which is "maximal" in the following sense:

$$
\text { for all } K \in \mathcal{I}(p, q) \text {, we can write } Y_{K}(x)=c_{K, I} Y_{I}(x) \text { with }\left|c_{K, I}\right| \leq 1 \text {. }
$$

The meaning of the maximality condition (3.4) is the following. Let $x \in M, p=p_{x}$ and $I \in$ $\mathcal{I}(p, q)$ which satisfies (3.4) at $x$. Choose any independent $p$-tuple $Y_{k_{1}}(x), \ldots, Y_{k_{p}}(x)$. Then $Y_{K}(x) \neq 0$ and (3.4) implies that $Y_{I}(x) \neq 0$ too. Therefore, $Y_{i_{1}}(x), \ldots, Y_{i_{p}}(x)$ are a basis of $\operatorname{span}\left\{Y_{i}(x): 1 \leq i \leq q\right\}$. Therefore, for all $k \in\{1, \ldots, q\}$, the solution $\left(b_{k}^{1}, \ldots, b_{k}^{p}\right) \in \mathbb{R}^{p}$ of the linear system $\sum_{\alpha=1}^{p} Y_{i_{\alpha}}(x) b_{k}^{\alpha}=Y_{k}(x)$ is unique and satisfies the estimate

$$
\left|b_{k}^{\alpha}\right| \leq 1 \text { for all } \alpha=1, \ldots, p .
$$


To see estimate (3.5), just note that, at the point $x$, given $k \in\{1, \ldots, q\}$ and $\alpha \in\{1, \ldots, p\}$,

$$
\begin{aligned}
Y_{i_{1}} \wedge \cdots \wedge Y_{i_{\alpha-1}} \wedge Y_{k} \wedge Y_{i_{\alpha+1}} \wedge \cdots \wedge Y_{i_{p}} & =Y_{i_{1}} \wedge \cdots \wedge Y_{i_{\alpha-1}} \wedge \sum_{\beta} b_{k}^{\beta} Y_{i_{\beta}} \wedge Y_{i_{\alpha+1}} \wedge \cdots \wedge Y_{i_{p}} \\
& =b_{k}^{\alpha} Y_{i_{1}} \wedge \cdots \wedge Y_{i_{p}}
\end{aligned}
$$

and the estimate follows, if $I$ satisfies (3.4) at $x$.

\subsection{Proof of Theorem 1.2}

Now we are in a position to get the proof of Theorem 1.2. Our arguments rely on some first-order expansion of a class of approximate exponential maps that was already discussed in [14]. We describe here the main steps with some comments on the uniform aspects of the estimates displaying in our setting, due to our global boundedness assumption $c_{i, j}^{k} \in L^{\infty}(M)$. Note that such uniformity can not be expected if $c_{i, j}^{k}$ are locally bounded only.

Let

$$
C_{1}:=\sup _{x \in M}\left|c_{i, j}^{k}(x)\right|<\infty \text { for all } i, j, k=1, \ldots, q
$$

Step 1. In order to denote appropriately the commutators, we use the following notation: given a word $w=w_{1} w_{2} \cdots w_{\ell}$ of length $\ell$ in the alphabet $\{1,2, \ldots, m\}$, we denote

$$
X_{w}=\left[X_{w_{1}},\left[X_{w_{2}}, \ldots,\left[W_{w_{\ell-1}}, X_{w_{\ell}}\right] \ldots\right]\right]
$$

Let $w_{1}, \ldots, w_{\ell} \in\{1, \ldots, m\}$ and define for $\tau \in \mathbb{R}$ the following $C^{1}$ diffeomorphisms from $M$ to itself (see also [10]):

$$
\begin{aligned}
C_{\tau}\left(X_{w_{1}}\right) & :=\exp \left(\tau X_{w_{1}}\right), \\
C_{\tau}\left(X_{w_{1}}, X_{w_{2}}\right) & :=\exp \left(-\tau X_{w_{2}}\right) \exp \left(-\tau X_{w_{1}}\right) \exp \left(\tau X_{w_{2}}\right) \exp \left(\tau X_{w_{1}}\right), \\
& \vdots \\
C_{\tau}\left(X_{w_{1}}, \ldots, X_{w_{\ell}}\right) & :=C_{\tau}\left(X_{w_{2}}, \ldots, X_{w_{\ell}}\right)^{-1} \exp \left(-\tau X_{w_{1}}\right) C_{\tau}\left(X_{w_{2}}, \ldots, X_{w_{\ell}}\right) \exp \left(\tau X_{w_{1}}\right) .
\end{aligned}
$$

Then, given the word $w=w_{1} w_{2} \cdots w_{\ell}$, define the approximate exponential of the commutator $X_{w}:=\left[X_{w_{1}},\left[X_{w_{2}}, \cdots,\left[W_{w_{\ell-1}}, X_{w_{\ell}}\right]\right]\right]$ by

$$
\mathrm{e}_{\mathrm{ap}}^{t X_{w_{1} w_{2} \ldots w_{\ell}}}:=\exp _{\mathrm{ap}}\left(t X_{w_{1} w_{2} \ldots w_{\ell}}\right):= \begin{cases}C_{t^{1 / \ell}}\left(X_{w_{1}}, \ldots, X_{w_{\ell}}\right), & \text { if } t \geq 0, \\ C_{|t|^{1 / \ell}}\left(X_{w_{1}}, \ldots, X_{w_{\ell}}\right)^{-1}, & \text { if } t<0 .\end{cases}
$$

Note that, since our vector fields are complete by assumption, $\exp _{\mathrm{ap}}\left(t X_{w_{1} w_{2} \ldots w_{\ell}}\right)$ is defined for all $t \in \mathbb{R}$.

Next, fix a word $w=w_{1} \cdots w_{\ell}$ and denote briefly $C_{t}:=C_{t}\left(X_{w_{1}}, \ldots, X_{w_{\ell}}\right)$. By [13, Theorem 3.5] and [14, Theorem 3.8], we have the expansions

$$
\frac{d}{d t} f\left(C_{t} x\right)=\ell t^{\ell-1} X_{w} f\left(C_{t} x\right)+\sum_{|v|=\ell+1}^{s} a_{v} t^{|v|-1} X_{v} f\left(C_{t} x\right)+t^{s} \sum_{|u|=1}^{s} b_{u}(x, t) X_{u} f\left(C_{t} x\right),
$$


and

$$
\begin{aligned}
\frac{d}{d t} f\left(C_{t}^{-1} x\right)= & -\ell t^{\ell-1} X_{w} f\left(C_{t}^{-1} x\right)+\sum_{|v|=\ell+1}^{s} \widetilde{a}_{v} t^{|v|-1} X_{v} f\left(C_{t}^{-1} x\right) \\
& +t^{s} \sum_{|u|=1}^{s} \widetilde{b_{u}}(x, t) X_{u} f\left(C_{t}^{-1} x\right),
\end{aligned}
$$

where $f: M \rightarrow \mathbb{R}$ is any $C^{2}$ test function. The sums on $v$ are empty if $|w|=s$. If not, we have the cancelation property $\sum_{|v|=\ell+1}\left(a_{v}+\widetilde{a}_{v}\right) X_{v}(x)=0$ for all $x \in M$. In our context, the estimates on $b_{u}$ and $\widetilde{b_{u}}$ are global as $x \in M$ :

$$
\left|b_{u}(x, t)\right|+\left|\widetilde{b_{u}}(x, t)\right| \leq C \text { for all } x \in M \quad \text { and } \quad|t| \leq 1 .
$$

Moreover, if the coefficients $c_{j k}^{\ell}$ are constant, $b_{u}$ and $\widetilde{b_{u}}$ are independent of $x$. This can be seen by tracking the details of the proof in [14, Theorem 3.8]. Note that our arguments can not provide any estimate on $b_{u}(x, t)$ for large $t$.

Step 2. Next we look at the adaptation to our setting of Theorem 3.11 in [14]. Under the hypotheses of Theorem 1.2, if $x \in M$, then the map $E_{x}$ introduced in (1.5) is globally defined (it is constructed by integral curves of the vector fields in $\mathcal{H}$ which are complete). Moreover $E_{x}$ is $C^{1}$ smooth (see [14, Theorem 3.11]). Actually $E_{x}$ sends points of the orbit $\mathcal{O}_{x}$ to points of $\mathcal{O}_{x}$. Finally, in terms of the homogeneous norm $\|h\|:=\max _{j}\left|h_{j}\right|^{1 / \ell_{j}}$, we have the first-order expansion for the differential (tangent map) $E_{*}$ of $E:=E_{x}$

$$
E_{*}\left(\partial_{h_{k}}\right)=Y_{k}(E(h))+\sum_{\ell_{j}=\ell_{k}+1}^{s} a_{k}^{j}(h) Y_{j}(E(h))+\sum_{j=1}^{q} \omega_{k}^{j}(x, h) Y_{j}(E(h)), \quad \text { for all } h \in \mathbb{R}^{q},
$$

where, for all $j, k, i$, we have the estimates

$$
\begin{aligned}
\left|a_{k}^{j}(h)\right| & \leq C\|h\|^{\ell_{j}-\ell_{k}} \quad \text { for all } h \in \mathbb{R}^{q} . \\
\left|\omega_{k}^{j}(x, h)\right| & \leq C\|h\|^{s+1-\ell_{k}} \text { for all } \quad x \in M \quad \text { and } h \in B_{\text {Euc }}(0,1) \subset \mathbb{R}^{q} .
\end{aligned}
$$

Here, the power-type functions $a_{k}^{j}$ are always independent of $x \in M$. An examination of the arguments in [14] shows that, under the hypotheses of Theorem $1.2\left(X_{j}\right.$ global and $\left.c_{j, k}^{\ell} \in L^{\infty}(M)\right)$, the functions $\omega(x, h)$ can be estimated uniformly as the base point $x$ lies in $M$. Precisely, the constant $C$ depends only on $C_{1}$ in (3.6). Finally, if the functions $c_{i, j}^{k}$ in (3.2) are constant (as it happens under the assumptions of Palais' Theorem), then $\omega_{k}^{j}$ depend on $h$ only (not on $x$ ) and the expansion (3.9) becomes completely independent of the base point $x$.

Step 3. Next we show inclusion (1.6), using (3.9). We claim that there is $C_{0}>1$ such that for all $x \in M$, the map $E_{x}: B_{\text {Euc }}\left(0, C_{0}^{-1}\right) \rightarrow\left(\mathcal{O}_{x}, g\right)$ satisfies the hypotheses of Theorem 1.1 [the metric $g$ is defined in (3.3)]. Let $h \in B_{\text {Euc }}(0,1) \subset \mathbb{R}^{q}$. Choose $I \in \mathcal{I}(p, q)$ which satisfies the maximality condition (3.4) at the point $E_{x}(h)$. Therefore for each $j \in\{1, \ldots, q\}$, we may write

$$
Y_{j}\left(E_{x}(h)\right)=\sum_{\alpha=1}^{p} b_{j}^{\alpha} Y_{i_{\alpha}}\left(E_{x}(h)\right)
$$


where by estimate (3.5) we have $\left|b_{j}^{\alpha}\right| \leq 1$ for all $\alpha$. Inserting into (3.9), we discover that the map $E_{x}=: E$ enjoys the following expansion for all $k \in\{1, \ldots, q\}$ :

$$
E_{*}\left(\partial_{h_{k}}\right)=: Y_{k}(E(h))+\sum_{\alpha=1}^{p} \chi_{k}^{\alpha} Y_{i_{\alpha}}(E(h)) \text { for all } h \in B_{\text {Euc }}(0,1) \subset \mathbb{R}^{q},
$$

where the functions $\chi_{k}^{\alpha}$ satisfy $\left|\chi_{k}^{\alpha}(x, h)\right| \leq C\|h\|$, for some universal constant $C$ depending on $C_{1}$ in (3.6) but not on $x \in M$. Specializing to $k=i_{\beta}$ with $\beta=1, \ldots, q$, we get

$$
E_{*}\left(\frac{\partial}{\partial h_{i_{\beta}}}\right)=Y_{i_{\beta}}(E(h))+\sum_{\alpha=1}^{p} \chi_{i_{\beta}}^{\alpha} Y_{i_{\alpha}}(E(h))=\sum_{\alpha=1}^{p}\left(\delta_{\beta}^{\alpha}+\chi_{i_{\beta}}^{\alpha}\right) Y_{i_{\alpha}}(E(h)) .
$$

In view of our estimate on the coefficients of the matrix $\chi=\left(\chi_{i_{\beta}}^{\alpha}\right)_{\alpha, \beta=1, \ldots, p}$, we can choose $r_{0}$ sufficiently small (independent of $x \in M$ ) so that that if $|h| \leq r_{0}$, then the operator norm of $\chi$ satisfies say $|\chi| \leq \frac{1}{2}$. Thus a Neumann series argument ensures that $\left|\left(I_{p}+\chi\right)^{-1}\right| \leq 2$ and ultimately we have shown that for all $h \in B_{\text {Euc }}\left(0, r_{0}\right) \subset \mathbb{R}^{q}$ and $\ell \in\{1, \ldots, q\}$, the system

$$
\sum_{j=1}^{q} E_{*}\left(\partial_{h_{j}}\right) \xi_{j}=Y_{\ell}(E(h))
$$

has a solution $\xi \in \mathbb{R}^{q}$ with $|\xi| \leq C_{0}$, where $C_{0}$ is an absolute constant. This shows that the assumptions of Theorem 1.1 are satisfied.

Proof of Corollary 1.3 Let $\gamma$ be a solution of (1.7). Assume without loss of generality that $\|b\|_{L^{\infty}} \leq 1$. Then we have

$$
x_{1}:=\gamma(\delta / 2) \in B_{\rho}(x, \delta) \subset E_{x}\left(B_{\text {Euc }}(0, \varepsilon)\right),
$$

by Theorem 1.2. Note that each map $E_{x}$ is $C^{1}$ on the whole $\mathbb{R}^{q}$. Thus, $x_{1}$ belongs to the bounded set $E_{x}(\{\|h\|<\varepsilon\})$. Next, let $x_{2}:=\gamma(\delta) \in B_{\rho}\left(x_{1}, \delta\right)$. Thus $x_{2}$ belongs to the bounded set $E_{x_{1}}\left(B_{\text {Euc }}(0, \varepsilon)\right)$. By iterating this argument for a sufficient number of times, say $v$ such that $\frac{\delta}{2} v \geq T$, we discover that the set $\gamma([0, T])$ is contained in a bounded set. Thus the solution $\gamma$ can not blow up.

\subsection{Examples}

In the first example we exhibit a pair of complete vector fields whose sum is not complete.

Example 3.1 Let $X=x y \partial_{x}$ and $Y=x y \partial_{y}$ in $\mathbb{R}^{2}$. Since

$$
\exp (t(X+Y))(1,1)=\left(\frac{1}{1-t}, \frac{1}{1-t}\right)
$$

then $X+Y$ is not complete.

In the following two examples, we exhibit finite-dimensional Lie algebras of vector fields arising in complex analysis.

Example 3.2 (CR vector fields on degenerate Siegel domains) Let $(x, t)=\left(x_{1}, x_{2}, t\right)$ be coordinates in $\mathbb{R}^{2} \times \mathbb{R}=\mathbb{R}^{3}$. Let $p(x):=|x|^{4}$ for $x=\left(x_{1}, x_{2}\right) \in \mathbb{R}^{2}$. Consider the pair of vector fields in $\mathbb{R}^{3}$

$$
\begin{aligned}
& X_{1}:=\partial_{x_{1}}+\partial_{2} p \partial_{t}=\partial_{x_{1}}+4 x_{2}|x|^{2} \partial_{t} \\
& X_{2}:=\partial_{x_{2}}-\partial_{1} p \partial_{t}=\partial_{x_{2}}-4 x_{1}|x|^{2} \partial_{t},
\end{aligned}
$$


where $\partial_{j}:=\frac{\partial}{\partial x_{j}}$. Here and in the sequel we use the notation $X_{j k}=\left[X_{j}, X_{k}\right], X_{i j k}=$ $\left[X_{i},\left[X_{j}, X_{k}\right]\right]$ and so on. We have $\operatorname{Lie}\left\{X_{1}, X_{2}\right\}=\operatorname{span}\left\{X_{1}, X_{2}, X_{12}, X_{112}, X_{212}, X_{1112}\right\}$.

Example 3.3 [CR vector fields on the sphere] We identify $\mathbb{R}^{4}$ with $\mathbb{C}^{2}$ via the identification $\mathbb{C}^{2} \ni\left(z_{1}, z_{2}\right) \simeq\left(x_{1}, x_{2}, y_{1}, y_{2}\right) \in \mathbb{R}^{2}$. Let

$$
\begin{aligned}
& X_{1}:=x_{1} \partial_{x_{2}}-y_{1} \partial_{y_{2}}-x_{2} \partial_{x_{1}}+y_{2} \partial_{y_{1}} \\
& X_{2}:=y_{1} \partial_{x_{2}}+x_{1} \partial_{y_{2}}-y_{2} \partial_{x_{1}}-x_{2} \partial_{y_{1}} .
\end{aligned}
$$

Then $X_{12}=2\left(y_{2} \partial_{x_{2}}-x_{2} \partial_{y_{2}}+y_{1} \partial_{x_{1}}-x_{1} \partial_{y_{1}}\right)$. We have $X_{112}=-4 X_{2}$ and $X_{212}=4 X_{1}$ so that $\operatorname{Lie}\left\{X_{1}, X_{2}\right\}=\operatorname{span}\left\{X_{1}, X_{2}, X_{12}\right\}$ has dimension three.

Finally we exhibit an example where the Lie algebra does not have finite dimension, but our assumption (1.3) holds, with globally bounded coefficients.

Example 3.4 Let again $(x, t)=\left(x_{1}, x_{2}, t\right) \in \mathbb{R}^{3}$, let $p\left(x_{1}, x_{2}, t\right)=\left(1+|x|^{2}\right)^{1 / 2}-1$ and take

$$
\begin{aligned}
& X_{1}=\partial_{1}+\left(\partial_{2} p\right) \partial_{t}=\partial_{1}+\left(1+|x|^{2}\right)^{-1 / 2} x_{2} \partial_{t} \\
& X_{2}=\partial_{2}-\left(\partial_{1} p\right) \partial_{t}=\partial_{2}-\left(1+|x|^{2}\right)^{-1 / 2} x_{1} \partial_{t} .
\end{aligned}
$$

Then

$$
\left[X_{1}, X_{2}\right]=-\frac{2+|x|^{2}}{\left(1+|x|^{2}\right)^{3 / 2}} \partial_{t} .
$$

The Hörmander's rank condition is fulfilled and moreover

$$
X_{112}=-\partial_{1} \frac{2+|x|^{2}}{\left(1+|x|^{2}\right)^{3 / 2}} \partial_{t}=\frac{\left(4+|x|^{2}\right) x_{1}}{\left(1+|x|^{2}\right)^{5 / 2}} \partial_{t} .
$$

We can write $X_{112}=: c(x) X_{12}$ with $c \in L^{\infty}\left(\mathbb{R}^{2}\right)$. The same holds for $X_{212}$.

Note that $\operatorname{Lie}\left\{X_{1}, X_{2}\right\}$ is not finite dimensional. This can be seen by looking at the behavior at infinity of the coefficients of higher-order commutators.

Acknowledgments The authors are members of the Gruppo Nazionale per l'Analisi Matematica, la Probabilità e le loro Applicazioni (GNAMPA) of the Istituto Nazionale di Alta Matematica (INdAM). The authors wish to thank the referee of the paper whose remarks have led to an improvement of the manuscript.

\section{References}

1. Abouqateb, A., Neeb, K.-H.: Integration of locally exponential Lie algebras of vector fields. Ann. Global Anal. Geom. 33(1), 89-100 (2008)

2. Chu, H., Kobayashi, S.: The automorphism group of a geometric structure. Trans. Amer. Math. Soc. 113, 141-150 (1964)

3. Earle, C.J., Eells, J.: Foliations and fibrations. J. Differ. Geom. 1, 33-41 (1967). (English)

4. Fefferman, C., Phong, D.H.: Subelliptic eigenvalue problems, Conference on harmonic analysis in honor of Antoni Zygmund, Vol. I, II (Chicago, Ill., 1981), Wadsworth Math. Ser., Wadsworth, Belmont, CA, 1983, pp. 590-606. MR 730094 (86c:35112)

5. Hermann, R.: A sufficient condition that a mapping of Riemannian manifolds be a fibre bundle. Proc. Am. Math. Soc. 11, 236-242 (1960)

6. Hermann, R.: The differential geometry of foliations. II. J. Math. Mech. 11, 303-315 (1962)

7. Hirschorn, R.M.: Controllability in nonlinear systems. J. Differ. Equ. 19(1), 46-61 (1975)

8. Hirsch, M., Smale, S., Devaney, R.: Differential equations, dynamical systems, and an introduction to chaos. Pure and applied mathematics, vol. 60, 2nd edn. Elsevier/Academic Press, Amsterdam (2004) 
9. Jouan, P.: Equivalence of control systems with linear systems on Lie groups and homogeneous spaces. ESAIM Control Optim. Calc. Var. 16(4), 956-973 (2010)

10. Lanconelli, E., Morbidelli, D.: On the Poincaré inequality for vector fields. Ark. Mat. 38(2), 327-342 (2000)

11. Lázaro-Camí, J.-A., Ortega, J.-P.: Superposition rules and stochastic Lie-Scheffers systems. Ann. Inst. Henri Poincaré Probab. Stat. 45(4), 910-931 (2009)

12. Leslie, J.A.: Some Frobenius theorems in global analysis. J. Differ. Geom. 2, 279-297 (1968)

13. Montanari, A., Morbidelli, D.: Nonsmooth Hörmander vector fields and their control balls. Trans. Am. Math. Soc. 364(5), 2339-2375 (2012)

14. Montanari, A., Morbidelli, D.: Almost exponential maps and integrability results for a class of horizontally regular vector fields. Potential Anal. 38(2), 611-633 (2013)

15. Manno, G., Oliveri, F., Vitolo, R.: On differential equations characterized by their Lie point symmetries. J. Math. Anal. Appl. 332(2), 767-786 (2007)

16. Michalska, H., Torres-Torriti, M.: A geometric approach to feedback stabilization of nonlinear systems with drift. Syst. Control Lett. 50(4), 303-318 (2003)

17. Nagel, A., Stein, E.M., Wainger, S.: Balls and metrics defined by vector fields. I. Basic properties. Acta Math. 155(1-2), 103-147 (1985)

18. Palais, Richard S.: A global formulation of the Lie theory of transformation groups, Mem. Amer. Math. Soc. No. 22, iii+123 (1957)

19. Patrick, J.: Rabier, global surjectivity of submersions via contractibility of the fibers. Trans. Am. Math. Soc. 347(9), 3405-3422 (1995)

20. Rabier, P.J.: Ehresmann fibrations and Palais-Smale conditions for morphisms of Finsler manifolds. Ann. Math. (2) 146(3), 647-691 (1997)

21. Varadarajan, V.S.: Lie groups, Lie algebras, and their representations, Graduate Texts in Mathematics, vol. 102, Springer-Verlag, New York, 1984, Reprint of the 1974 edition 